\title{
Effects of multiple firings on the microstructure of zirconia and veneering ceramics
}

\author{
Murat ALKURT' ${ }^{1}$, Zeynep YEŞIL DUYMUS ${ }^{1}$ and Mustafa GUNDOGDU² \\ ${ }^{1}$ Department of Prosthodontics, Faculty of Dentistry, Recep Tayyip Erdogan University, Rize, Turkey \\ ${ }^{2}$ Department of Prosthodontics, Faculty of Dentistry, Atatürk University, Erzurum, Turkey \\ Corresponding author, Murat ALKURT; E-mail: muratalkurt@hotmail.com
}

\begin{abstract}
The aim of study was to evaluate the effects of multiple firings on the microstructures of zirconia and two ceramics. Vita VM9 (VMZ) and Cerabien ZR (C-Z) ceramics on a zirconia framework and zirconia without veneering ceramic (WO-Z) were evaluated. Firing methods included firing two, five, and ten times $(n=10)$. The effects of multiple firings on the surface hardness of the materials were evaluated using a Vickers hardness (HV) tester. Data were analyzed by two-way ANOVA and Tukey's test $(\alpha=0.05)$. After firing five and ten times, the hardness of VM-Z and C-Z increased significantly $(p<0.001)$. The HVs of the Cerabien ZR and Vita VM9 veneering ceramics were similar $(p>0.05)$. In the XRD analysis, zirconia had similar tetragonal $(\mathrm{t})$-monoclinic $(\mathrm{m})$ phase transformations of Y-TZP after the different firing times. Clinically, multiple firings did not affect the microstructure of zirconia, but the structures of the two ceramics were affected.
\end{abstract}

Keywords: Dental materials, XRD, Vickers hardness test

\section{INTRODUCTION}

Computer-aided design/computer-aided manufacturing $(\mathrm{CAD} / \mathrm{CAM})$ techniques are widely used in zirconia frameworks of crowns and fixed partial dentures. With the rise of $\mathrm{CAD} / \mathrm{CAM}$ technology, yttria-stabilized tetragonal zirconia polycrystals in the pre-sintered state (Y-TZP) have become a popular material ${ }^{1,2)}$. Pre-sintered zirconia frameworks must be fired in accordance with the manufacturers' firing temperatures ${ }^{3}$. After the firing protocol, the sintered zirconia framework is covered with veneer porcelain for a natural esthetic appearance.

Zirconia is a polymorphic material that exists in three crystal structures: monoclinic (from room temperature to $\left.1,170^{\circ} \mathrm{C}\right)$, tetragonal $\left(1,170-2,370^{\circ} \mathrm{C}\right)$, and cubic (above $\left.2,370^{\circ} \mathrm{C}\right)^{1,4)}$. Sintered Y-TZP has a nearly $100 \%$ tetragonal microstructure. However, zirconia has exhibited reverse transformation, from the tetragonal to the monoclinic phase, at lowtemperature degeneration. This phase transformation has led to increased fracture strength in clinically applied Y-TZP ${ }^{5,6}$. Previous studies have evaluated the correlation between heat treatment and the monoclinicto-tetragonal phase transformation. Collectively, these studies have suggested that the monoclinic-to-tetragonal phase transformation of Y-TZP may be triggered by heat treatment during the veneering procedure ${ }^{7-9)}$. However, the effect of multiple firings on phase transformation in zirconia has not been investigated.

Porcelain ceramic is usually veneered on the Y-TZP core by using sintering or hot-pressing techniques. The chemical property of ceramic materials, which consist mainly of various crystal phases, is affected by a variety

Color figures can be viewed in the online issue, which is available at J-STAGE.

Received Dec 15, 2015: Accepted May 29, 2016

doi:10.4012/dmj.2015-429 JOI JST.JSTAGE/dmj/2015-429 of factors, such as the composition and microstructure of the ceramic materials, as well as temperature ${ }^{10-13)}$. Firing protocols performed at high temperature (750 $900^{\circ} \mathrm{C}$ ) usually apply $2-5$ successive firings in simple cases. However, in complicated cases, ten firings are sometimes required ${ }^{11)}$.

Previous studies have investigated the effects of the veneering firing protocol on the surface hardness of porcelain. Tang et al. ${ }^{14)}$ and El-Kheshen and Zawrah ${ }^{15)}$ reported that multiple firings increased the density and decreased the porosity of porcelain and increased its surface hardness. Thus, the effects of firing times and temperatures on the properties of ceramics and zirconia cannot be ignored.

The purpose of this study was to evaluate the effects of multiple firings on the microstructures of zirconia and veneering ceramics. The authors hypothesized that 1) multiple firing causes the tetragonal-to-monoclinic phase transformation of Y-TZP and 2) there are no differences in the surface hardness of zirconia and veneering ceramics after multiple firings.

\section{MATERIALS AND METHODS}

The materials used in this study are presented in Table 1. Two different veneering ceramics on zirconia framework - Vita VM9 (VM-Z) and Cerabien ZR (C-Z) and zirconia without veneering ceramic (WO-Z) were evaluated (Table 2 and Fig. 1).

Ninety disc-shaped zirconia specimens $(15 \mathrm{~mm}$ diameter $\times 1.3 \mathrm{~mm}$ thickness) fabricated according to ISO 6872 were sintered to full density in a furnace according to the manufacturer's instructions. After the sintering process, the zirconia specimens were divided into three groups: VM-Z, C-Z, and WO-Z $(n=30)$. For the 
Table 1 Codes, manufacturing of materials and chemical composition

\begin{tabular}{lccc}
\hline Materials & Codes & Manufacturer & Chemical composition \\
\hline Zirconia & $\mathrm{Z}$ & 3M ESPE Dental, Seefeld, Germany & $\mathrm{ZrO}_{2}(97 \%), \mathrm{Y}_{2} \mathrm{O}_{3}(3 \%)$. \\
Cerabien ZR & $\mathrm{C}$ & Noritake, Nagoya, Japan & Potassium aluminosilicate glass, leucite, etc. \\
Vita VM9 & $\mathrm{VM}$ & Zahnfabrik, Bad Säckingen, Germany & Feldspar, alumina, cerium oxide, leucite, etc. \\
\hline
\end{tabular}

Table 2 Descriptions of groups

\begin{tabular}{lc}
\hline Codes & Groups \\
\hline WO-Z & Without veneering ceramic Zr framework \\
C-Z & Zr framework with Cerabien ZR ceramic \\
VM-Z & Zr framework with VM9 ceramic \\
\hline
\end{tabular}

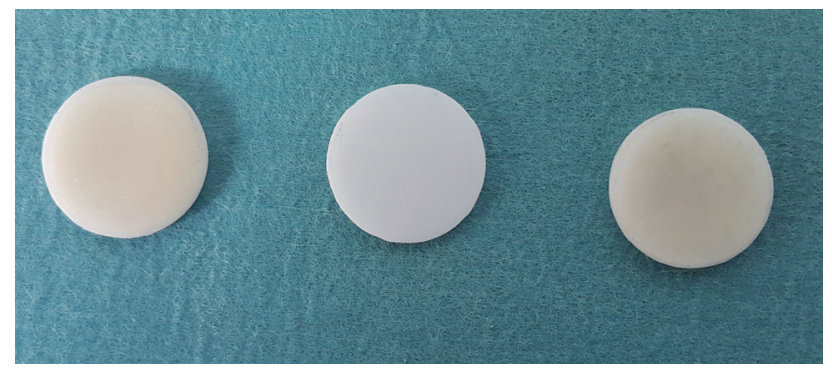

Fig. 1 VM-Z, WO-Z and C-Z specimen.

VM-Z and C-Z group specimens, using a stainless steel mold (Fig. 2), a 1.0 mm-thick Vita VM9 or Cerabien ZR ceramic porcelain layer was applied to the surface of the zirconia framework according to the manufacturer's recommendations (Table 3 ). The VM-Z and C-Z bilayered specimens were mirror-polished to a final thickness of $1.00( \pm 0.13) \mathrm{mm}$ using a polishing machine (Phoenix Beta, Buehler, Lake Bluff, IL, USA). Then, the VM-Z and C-Z groups were divided into three firing time subgroups: two, five, and ten times $(n=10)$. No porcelain layer was applied to the remaining 30 zirconia framework specimens (WO-Z), which were also divided three firing time sub-groups: two, five, and ten times $(n=10)$.

\section{Surface hardness}

To evaluate the influence of multiple firings on surface hardness, a Vickers hardness tester (TMTeck HV, Beijing, China) was used, with a load of $19.61 \mathrm{~N}$ and a dwell time of $20 \mathrm{~s}$ for the VM-Z and C-Z groups and a load of $98.07 \mathrm{~N}$ and a dwell time of $15 \mathrm{~s}$ for the WO-Z group. Vickers microhardness tests were performed as the average of ten indents per specimen. In the VM-Z and C-Z groups, the Vickers microhardness tests were performed on the polished surface, and cracks generated along the indentation diagonal were measured. In the WO-Z group, a Vickers indenter was pressed into the

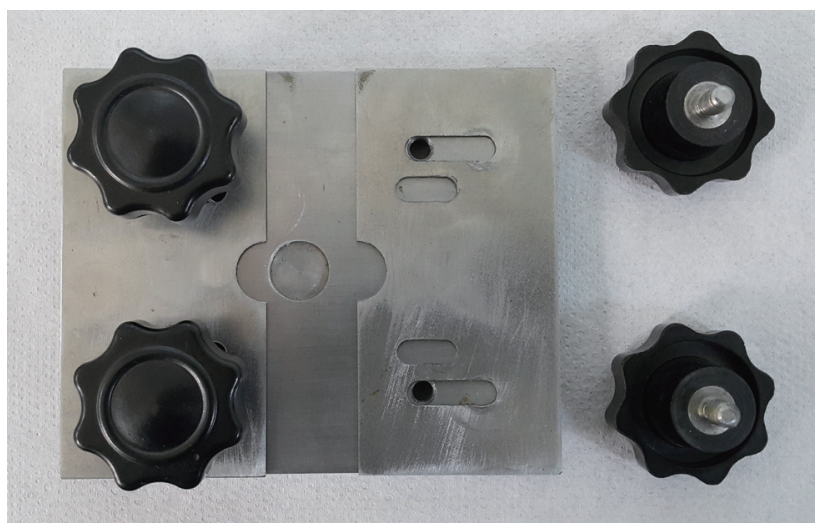

Fig. 2 Stainless steel mold.

surface of the polished specimen and the diagonal was measured. Then, all of the measurements converted to the Vickers hardness (HV) value.

\section{Phase transformation}

In the three firing times of the WO-Z group, three specimens were chosen according to surface harness value: close to maximum, minimum, and average for the group. Tetragonal (t)-monoclinic (m) phase transformations of Y-TZP were analyzed with a Rigaku SmartLab system (Rigaku America, The Woodlands, TX, USA) using $\mathrm{Cu} \mathrm{K} \alpha_{1}$ X-rays. The X-ray diffraction (XRD) profiles were acquired from 25 to $37^{\circ}(2 \theta)$ with a step size of 0.001 and $2.0156 \mathrm{deg} / \mathrm{min}$.

The phase transformation calculations were based on the Garvie-Nicholson method using the formula ${ }^{16)}$ :

$\left.\mathrm{X}_{\mathrm{m}}=[\operatorname{Im}(-111)+\operatorname{Im}(111)] /[\operatorname{Im}(-111)+\operatorname{Im}(111)]+\operatorname{It}(101)\right]$

where $\mathrm{X}_{\mathrm{m}}$ is the mass fraction of the monoclinic phase, $\operatorname{Im}(-111)$ is the intensity of the monoclinic peak at $28.2^{\circ}, \operatorname{Im}(111)$ is the intensity of the monoclinic peak at $31.5^{\circ}$, and $\operatorname{It}(101)$ is the intensity of the monoclinic peak at $30.2^{\circ}$. 
Table 3 Firing schedules of layering porcelains

\begin{tabular}{lcr}
\hline Porcelain materials & Cerabien ZR & Vita VM9 \\
\hline Preheating $\left({ }^{\circ} \mathrm{C}\right)$ & 600 & 500 \\
Drying (min) & 5 & 6 \\
Raise of temp $\left({ }^{\circ} \mathrm{C} / \mathrm{min}\right)$ & 45 & 55 \\
Start vacuum $\left({ }^{\circ} \mathrm{C}\right)$ & 600 & 500 \\
Final vacuum $\left({ }^{\circ} \mathrm{C}\right)$ & 960 & 910 \\
Final temp $\left({ }^{\circ} \mathrm{C}\right)$ & 960 & 910 \\
Holding time $(\min )$ & 1 & 1 \\
Cooling time (min) & 4 & 4 \\
\hline
\end{tabular}

Table 4 Mean surface hardness values and standard deviations

\begin{tabular}{clcccc}
\hline Test method & Group & 2 times & 5 times & 10 times & Mean \\
\hline HV test with 98.07 N and 15 s & WO-Z & $1,041.44 \pm(107.07)^{\mathrm{a}}$ & $1,028.73 \pm(86.31)^{\mathrm{a}}$ & $940.11 \pm(100.54)^{\mathrm{a}}$ & $1,003.42 \pm(105.07)$ \\
& C-Z & $363.84 \pm(47.99)^{\mathrm{Aa}}$ & $486.47 \pm(44.63)^{\mathrm{Ab}}$ & $545.82 \pm(21.20)^{\mathrm{Ac}}$ & $465.37 \pm(86.09)$ \\
HV test with 19.61 N and 20 s & VM-Z & $383.55 \pm(56.35)^{\mathrm{Aa}}$ & $495.59 \pm(33.58)^{\mathrm{Ab}}$ & $558.17 \pm(21.32)^{\mathrm{Ac}}$ & $479.10 \pm(82.91)$ \\
\hline
\end{tabular}

For HV test with $98.07 \mathrm{~N}$ and $15 \mathrm{~s}$, lowercase indicate that significant difference of firing times. For HV test with $19.61 \mathrm{~N}$ and $20 \mathrm{~s}$, capital letters indicate that statically significant difference of porcelain material; lowercase indicate that significant difference of firing times.

Table 5 Two-way ANOVA (veneering ceramic) for Vikers hardness test

\begin{tabular}{lrrrrr}
\hline \multicolumn{1}{c}{ Source } & Sum of Squares & $d f$ & Mean Square & F & $p$ \\
\hline Porcelain materials & $2,188.257$ & 1 & $2,188.257$ & 1.374 & 0.246 \\
Firing times & $326,043.360$ & 2 & $163,021.680$ & 102.345 & $0.000^{*}$ \\
Porcelain materials×firing times & 440.271 & 2 & 220.136 & 0.138 & 0.871 \\
Error & $86,014.595$ & 54 & $1,592.863$ & - & - \\
Total & $1.380 \mathrm{E} 7$ & 60 & - & - & - \\
Corrected total & $417,149.604$ & 59 & - & - \\
\hline
\end{tabular}

* statistically different values

Statistical analyses

Two variables of the veneering ceramics and their interactions were investigated: porcelain materials and firing times. The data were analyzed using two-way ANOVA, and pairwise comparisons were performed with Tukey's test.

The effects of the firing time variable were investigated in the zirconia without veneering ceramic. The surface hardness value was analyzed using one-way ANOVA, and pairwise comparisons were performed with Tukey's test. The level of statistical significance was set at 0.05 for all analyses (version 16, SPSS, Chicago, IL, USA).

\section{RESULTS}

The mean surface hardness values and standard deviations for each group are presented in Table 4. For the veneering ceramics, the results of the two-way ANOVA (Table 5) and Tukey's tests showed that firing times significantly affected surface hardness $(p<0.001)$. The HVs of VM-Z and C-Z increased significantly after five and ten firings $(p<0.05)$. The HVs of the Cerabien ZR and Vita VM9 veneering ceramics were similar $(p>0.05)$.

For the zirconia without veneering ceramic, the results of the one-way ANOVA (Table 6) and Tukey's 
Table 6 One-way ANOVA (zirconia without veneering ceramic) for Vikers hardness test

\begin{tabular}{lccccc}
\hline & Sum of Squares & $d f$ & Mean Square & F & $p$ \\
\hline Between Groups & $54,851.745$ & 2 & $27,425.873$ & 2.835 & -0.078 \\
Within Groups & $232,202.751$ & 27 & $9,675.115$ & - & - \\
Total & $287,054.496$ & 29 & - & - \\
\hline
\end{tabular}
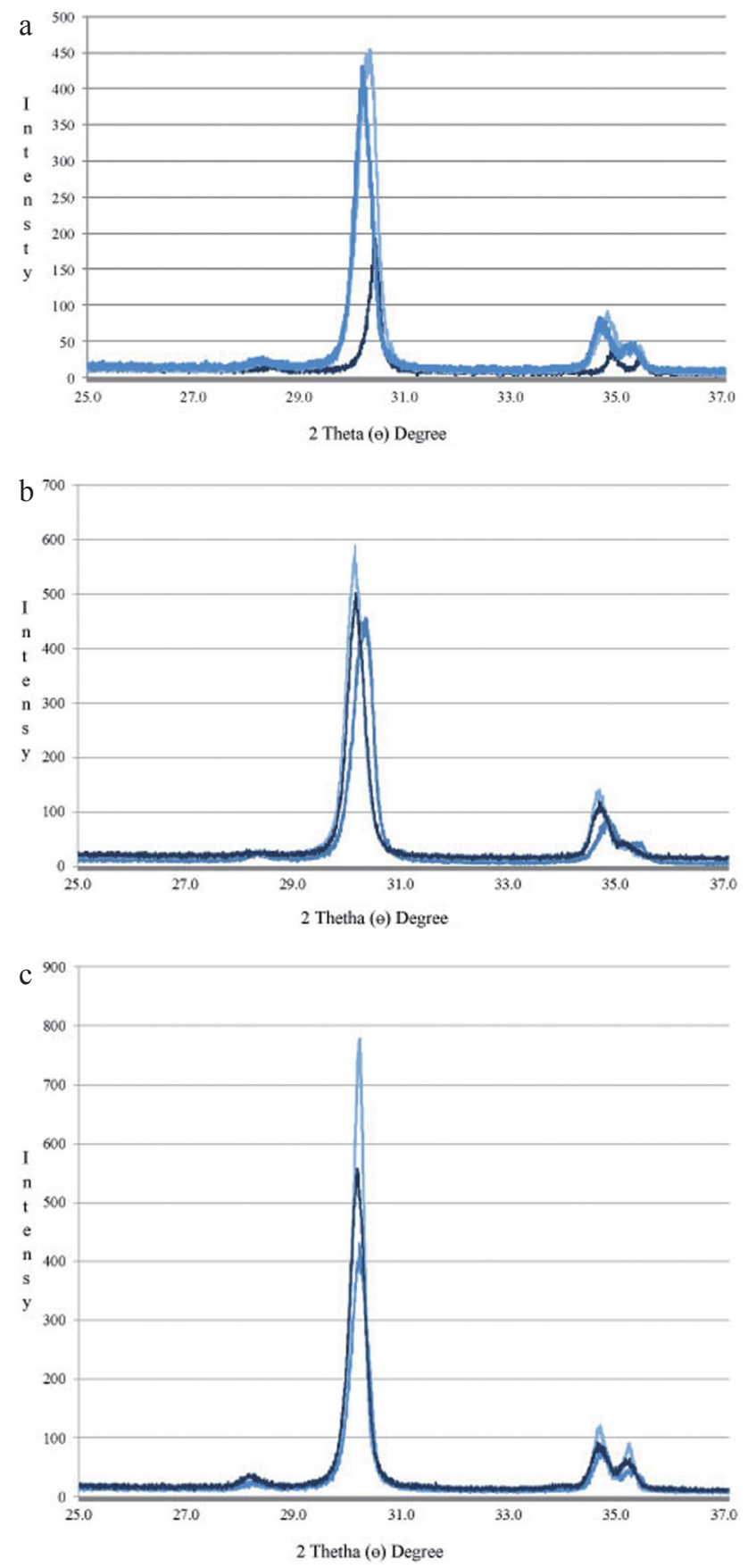

Fig. 3 a: XRD analysis of 2 times firing. b: XRD analysis of 5 times firing. c: XRD analysis of 10 times firing. test showed that firing times did not significantly affect surface hardness $(p>0.05)$. The $\mathrm{HV}$ of the zirconia decreased after five and ten firings, and the lowest $\mathrm{HVs}$ were found after ten firings. However, these differences were not statically significant $(p>0.05)$.

The XRD analysis of each group is presented in Figs. 3a-c. In the XRD analysis, the ranges of the relative amounts of monoclinic phase were as follows: two firings, 8-14\%; five firings, 8-9\%; and ten firings, $5-9 \%$. The highest relative amount of monoclinic phase (14\%) was observed after two firings, and the lowest (5\%) was observed after ten firings.

\section{DISCUSSION}

Based on the results of this study, the first hypothesis, stating that multiple firings cause tetragonal-tomonoclinic phase transformation of Y-TZP, was rejected. The second hypothesis, stating that there is no significant difference in the surface hardness of veneering ceramics after multiple firings, was also rejected. However, this hypothesis regarding surface hardness was accepted in the case of zirconia without veneering ceramic.

The HV test is a method of calculating the surface hardness of a material by indentation using a Vickers indenter. The researchers used different test conditions, including test load and loading time. The HVs cannot calculate accurately when the load is too heavy or too light ${ }^{17-19)}$. In scanning electron microscopy images of zirconia, Harada et al. ${ }^{20)}$ observed that cracks and the surface change, especially at 196 and 294 N. However, higher loads would cause catastrophic crack propagation around the indentation diagonal in ceramic materials. Therefore, an optimal indentation load and time should be selected to measure the surface hardness of materials. In this study, a load of $19.61 \mathrm{~N}$ and a dwell time of $20 \mathrm{~s}$ for the ceramic groups and a load of $98.07 \mathrm{~N}$ and a dwell time of $15 \mathrm{~s}$ for zirconia were used.

In this study, the results of the multiple firings of zirconia indicated that surface hardness was lower in the five and ten firings groups than in the two firings group. It was also observed that there was a correlation between higher number of firings and decreased surface hardness in zirconia. However, this correlation did not affect the surface hardness of zirconia statically. The correlation between number of firings and surface hardness of zirconia was in agreement with the results of previous studies. Kanno et al. ${ }^{21)}$ observed that total average microhardness was significantly lower after firing than before firing, with a difference of $2 \%$. 
Zirconia frameworks are usually fabricated using partially sintered Y-TZP blocks that undergo a sintering process to convert to fully sintered Y-TZP. After the sintering process, conventional porcelain is applied to Y-TZP at temperatures up to $750-900^{\circ} \mathrm{C}^{14)}$. Several authors have reported that these heat treatments induce a monoclinic-to-tetragonal phase transformation ${ }^{6,9)}$.

In previous studies, a maximum $25 \%$ monoclinic phase in zirconia was considered an acceptable level ${ }^{22,23)}$. In this study, the range of relative amount of monoclinic phase was $5-14 \%$. The XRD analysis results showed that multiple firings did not change the amount of monoclinic phases significantly. The greatest content of monoclinic phase was observed after two firings (14\%), followed by five (9\%) and ten (5\%) firings. This study agrees with previous studies that heat treatment decreased the content of the monoclinic phase $\mathrm{e}^{6,9}$. Kosmac et $a l .{ }^{6}$ ), who applied a heat treatment of $900^{\circ} \mathrm{C}$ and holding time of one hour, and Guazzato et al..$^{9}$, who applied a heat treatment of $930^{\circ} \mathrm{C}$ and holding time one min, concluded that the monoclinic-to-tetragonal phase transformation might occur after heat treatment.

Cattani-Lorente et $a l .{ }^{24)}$, Alghazzawi et $a l .{ }^{25)}$, Catledge et al. ${ }^{26)}$ and Santos et al. ${ }^{27)}$ found a strong linear correlation between the increase in monoclinic fraction and decrease in surface hardness. However, no linear correlation between amount of monoclinic phase and surface hardness was observed in the current study.

Hardness, which affects the finishability, polishability, and occlusal wear resistance of a material, is an important property ${ }^{28}$. Materials with a high degree of hardness exhibit greater abrasion resistance and are difficult to polish. Veneering ceramics, which become harder and denser with multiple firings ${ }^{29)}$, may have the advantage of improved resistance to prevent material failures ${ }^{30}$.

In simple cases, conventional porcelains usually undergo a $2-5$ successive firings. This protocol can sometimes be as high as ten 10 firings if the dentist needs shape and color corrections. In this study, the HVs of the layering porcelains were compared, and there were significant differences in the surface hardnesses of the veneering ceramics before and after undergoing multiple firings. Increasing the number of firings increased the surface hardness of the layering porcelains. This observed result was in accordance with the results of a previous study by Tang et al. ${ }^{14)}$, who found that multiple firings increased the density and surface hardness and decreased the porosity of porcelain. The increase in surface hardness might be related to the densification mechanism of liquid-phase sintering in porcelain ${ }^{15)}$. El-Kheshen and Zawrah ${ }^{15)}$ observed that a composite of glass/ceramic densified with increased firing temperature.

The limitations of this study are that only two porcelain ceramics and surface hardness properties were evaluated. Further studies are recommended to evaluate the effects of multiple firings on other properties, such as color and translucency.

\section{CONCLUSIONS}

Based on the results of this study, the following could be conclusions can be made:

- Multiple firings affect the surface hardness of veneering ceramics significantly.

- Multiple firings do not cause tetragonal-tomonoclinic phase transformations or statistically significant changes in surface hardness in zirconia.

\section{ACKNOWLEDGMENTS}

The investigation was supported in part by Scientific Research Projects Committee of Atatürk University, Erzurum (Project no: 2011/70).

\section{REFERENCES}

1) Piconi C, Maccauro G. Zirconia as a ceramic biomaterial. Biomaterials 1999; 20: 1-25.

2) Ban S. Reliability and properties of core materials for all ceramic dental restorations. J Dent Sci Rev 2008; 44: 3-21.

3) Ban S, Okuda Y, Noda M, Tsuruki J, Kawai T, Kono H. Contamination of dental zirconia before final firing: effects on mechanical properties. Dent Mater J 2013; 32: 1011-1019.

4) Denry I, Kelly JR. State of the art of zirconia for dental applications. Dent Mater 2008; 24: 299-307.

5) Denry IL, Holloway JA. Microstrucrural and crystallographic surface changes after grinding zirconia-based dental ceramics. J Biomed Mater Res B: Appl Biomater 2006; 76: 440-448.

6) Kosmac T, Oblak C, Marion L: The effects of dental grinding and sandblasting on ageing and fatigue behavior of dental zirconia (Y-TZP) ceramics. J Eur Ceram Soc 2008; 28: 10851090.

7) Sato H, Yamada K, Pezzotti G, Nawa M, Ban S. Mechanical properties of dental zirconia ceramics changed with sandblasting and heat treatment. Dent Mater J 2008; 27: 408-414.

8) Kosmac T, Oblak C, Jevnikar P, Funduk N, Marion L. Strength and reliability of surface treated Y-TZP dental ceramics. J Biomed Mater Res 2000; 53: 304-313.

9) Guazzato M, Quach L, Albakry M, Swain MV. Influence of surface and heat treatments on theflexural strength of Y-TZP dental ceramic. J Dent 2005; 33: 9-18.

10) Kukiattrakoon B, Hengtrakool C, Kedjarune-Leggat U. Degradability of fluorapatite-leucite ceramics in naturally acidic agents. Dent Mater J 2010; 29: 502-511.

11) Ccahuana VZ, Ozcan M, Mesquita AM, Nishioka RS, Kimpara ET, Bottino MA. Surface degradation of glass ceramics after exposure to acidulated phosphate fluoride. J Appl Oral Sci 2010; 18: 155-165.

12) Lynch ME, Folz DC, Clark DE. Use of FTIR reflectance spectroscopy to monitor corrosion mechanisms on glass surface. J Non Cryst Solids 2007; 353: 2667-2674.

13) Kim MJ, Kim YK, Kim KH, Kwon TY. Shear bond strengths of various luting cements to zirconia ceramic: surface chemical aspects. J Dent 2011; 39: 795-803.

14) Tang X, Nakamura T, Usami H, Wakabayashi K, Yatani H. Effects of multiple firings on the mechanical properties and microstructure of veneering ceramics for zirconia frameworks. J Dent 2012; 40: 372-380.

15) El-Kheshen AA, Zawrah MF. Sinterability, microstructure and properties of glass/ceramic composites. Ceram Int 2003; 29: 251-257.

16) Garvie RC, Nicholson PS. Phase analysis in zirconia systems. 
J Am Ceram Soc 1972; 55: 303-330.

17) Anstis GR, Chantikul P, Lawn BR, Marshall DB. A critical evaluation of indentation techniques for measuring fracture toughness, I. Direct crack measurements. J Am Ceram Soc 1981; 64: 533-538.

18) Niihara K, Morena R, Hasselman DPH. Evaluation of KIc of brittle solids by the indentation method low-to-indent ration. Mater Sci Lett 1982; 1: 13-19.

19) Okada T, Shinya A, Yokozuka S. Effects of loading time on fracture toughness with indentation method. Shigaku 1990; 78: 460-486.

20) Harada K, Shinya A, Yokoyama D, Shinya A. Effect of loading conditions on the fracture toughness of zirconia. $J$ Prosthodont Res 2013; 57: 82-87.

21) Kanno T, Milleding P, Wennerberg A. Topography, microhardness, and precision of fit on ready-made zirconia abutment before-after sintering process. Clin Implant Dent Relat Res 2007; 9: 156-165.

22) ISO Standard 13356:2008. Implants for surgery —Ceramic materials based on yttria-stabilized tetragonal zirconia (Y-TZP).

23) Lughi V, Sergo V. Low temperature degradation -aging- of zirconia: a critical review of the relevant aspects in dentistry. Dent Mater 2010; 26: 807-820.
24) Cattani-Lorente M, Scherrer SS, Ammann P, Jobin M, Wiskott HW. Low temperature degradation of a Y-TZP dental ceramic. Acta Biomater 2011; 7: 858-865.

25) Alghazzawi TF, Lemons J, Liu PR, Essig ME, Bartolucci AA, Janowski GM. Influence of low-temperature environmental exposure on the mechanical properties and structural stability of dental zirconia. J Prosthodont 2012; 21: 363-369.

26) Catledge SA, Cook M, Vohra YK, Santos EM, McClenny MD, David Moore K. Surface crystalline phases and nanoindentation hardness of explanted zirconia femoral heads. J Mater Sci Mater Med 2003; 14: 863-867.

27) Santos EM, Vohra S, Catledge SA, Mec Clenny D, Lemons JE, Moore KD. Examination of surface and material properties of explanted zirconia femoral heads. J Arthroplasty 2004; 19: 30-34.

28) Charlton DG, Roberts HW, Tiba A. Measurement of select physical and mechanical properties of 3 machinable ceramic materials. Quintessence Int 2008; 39: 573-579.

29) Hildebrand HF, Veron C, Martin P. Nickel, chromium, cobalt dental alloys and allergic reactions: an overview. Biomaterials 1989; 10: 545-548.

30) Oh WS, Delong R, Anusavice KJ. Factors affecting enamel and ceramic wear: a literature review. J Prosthet Dent 2002; 87: 451-459. 